\title{
BMJ Open Efficacy of low-magnitude high- frequency vibration (LMHFV) on musculoskeletal health of participants on wheelchair: a study protocol for a single-blinded randomised controlled study
}

\author{
Simon Kwoon Ho Chow, Chung Yan Ho, Hiu Wun Wong, Yu Ning Chim (1) , \\ Ronald Man-Yeung Wong, Wing Hoi Cheung (c)
}

To cite: Chow SKH, Ho CY, Wong HW, et al. Efficacy of low-magnitude highfrequency vibration (LMHFV) on musculoskeletal health of participants on wheelchair: a study protocol for a single-blinded randomised controlled study. BMJ Open 2020;10:e038578. doi:10.1136/ bmjopen-2020-038578

- Prepublication history and additional material for this paper are available online. To view these files, please visit the journal online (http://dx.doi. org/10.1136/bmjopen-2020038578).

Received 18 March 2020 Revised 29 0ctober 2020 Accepted 12 November 2020

A) Check for updates

(C) Author(s) (or their employer(s)) 2020. Re-use permitted under CC BY-NC. No commercial re-use. See rights and permissions. Published by BMJ.

Department of Orthopaedics and Traumatology, The Chinese University of Hong Kong, New Territories, Hong Kong

Correspondence to Professor Wing Hoi Cheung; louischeung@cuhk.edu.hk

\section{ABSTRACT}

Background 0steoporosis is an age-related disease with progressive loss of bone, leading to fragile bone. It is one of the major health issues in older adults and causes medical, social and economic impacts globally. Patients with osteoporosis have high risk of osteoporotic fractures. Low-magnitude high-frequency vibration (LMHFV) is a non-invasive biophysical intervention providing wholebody mechanical stimulation. Previous studies showed that LMHFV is beneficial to muscle strength, postural control, balancing ability, new bone formation, spinal bone mineral density (BMD) and blood circulation. During the LMHFV treatment, older adults need to stand upright on the platform for $20 \mathrm{~min} /$ day. However, some physically weak elderlies with poor musculoskeletal ability cannot stand for a long period. Therefore, the design of vibration platform is modified for the disabled patients to treat at sitting position and the efficacy of LMHFV on this group of elderlies will be verified. It is hypothesised that new design of LMHFV is beneficial to wheelchair users in terms of vertebral BMD, muscle health and musculoskeletal functions.

Methods This study is a single-blinded randomised controlled trial to investigate the effect of LMHFV on vertebral BMD, muscle health, balancing ability and functional ability in wheelchair users (mainly on wheelchair for outdoor activities). Healthy elderlies aged 65 years or above with walking difficulties and using wheelchair are eligible. Exclusion criteria are those: (1) who cannot stand and walk independently, (2) who have vibration treatment before, (3) with malignancy, (4) with acute fractures or severe osteoarthritis, (5) with cardiovascular concern such as with pacemaker in situ, (6) with chronic inflammatory conditions known to affect muscle metabolism such as rheumatoid arthritis and (7) with high frequency of physical activities, such as participants who participated in regular exercise five times a week or more. Recruited participants will be randomised to either LMHFV or control group. Participant assigned to LMHFV group will receive $\mathrm{LMHFV}(35 \mathrm{~Hz}, 0.3 \mathrm{~g}$ (g=gravitational acceleration), $20 \mathrm{~min} /$ day, at least three times/week) for 6 months. The primary outcome is BMD at the lumbar spine to be
Strengths and limitations of this study

- This is the first study that investigates the efficacy of low-magnitude high-frequency vibration (LMHFV) on wheelchair users, hence data generated will be with high clinical translation values.

- This trial cannot be double-blinded since blinding subjects for the LMHFV treatment is not feasible.

- The vibration signals can be easily felt and placebo is rare in vibration studies.

assessed by dual-energy X-ray absorptiometry that is clinically recommended for the diagnosis of osteoporosis. All primary and secondary outcome assessments for all groups will be performed in the investigators' institute at baseline and 6 months post treatment.

Discussion This study aims to investigate the effects of LMHFV on wheelchair users. The findings of this study will help to confirm the efficacy of LMHFV on vertebral $\mathrm{BMD}$, muscle health, balancing ability and functional outcomes in wheelchair using elderlies. LMHFV therapy is an intervention strategy that is easy to implement at the community healthcare level or individually at home that has previously been proven to reduce fall risk and muscle strength at the lower limb. The ultimate goal is to improve their bone and muscle quality of wheelchair users, as well as enhancing their quality of life.

Trial registration number ClinicalTrials.gov (NCT04180267).

\section{BACKGROUND}

Ageing is an emerging socioeconomic problem in Hong Kong. The ageing population at 65 years or above increased continuously from 0.46 million $(8.2 \%)$ to 1.27 million (17.9\%) from 1988 to $2018 .{ }^{1}$ The prevalence of osteoporosis also increases due to the escalating ageing population. ${ }^{2}$ Osteoporosis is an age-induced disorder with progressive loss 
of bone that leads to deterioration of bone microarchitecture and hence low bone mineral density (BMD). It increases the risk of fragility fracture known to be associated with increased morbidity and mortality. ${ }^{3}$

Older adults with disability or mobility impairments due to trauma, chronic illnesses or overweight may avoid walking and rely on wheelchairs for most daily activities. Many studies showed that wheelchair users had greater bone loss compared with walkers. ${ }^{45}$ Physical activity is one of the recommended approaches for preventing osteoporosis. However, some older adults may be restricted from exercise training because they are not physically fit to perform the intensive exercise, ${ }^{6}$ leading to a vicious cycle of musculoskeletal deterioration.

Low-magnitude high-frequency vibration (LMHFV) is a promising intervention proposed as an alternative to physical exercise ${ }^{7}$ for treating osteoporosis. It is a non-invasive biophysical intervention that provides systemic vertical vibrations at $20-90 \mathrm{~Hz}$ at amplitude of less than $1.0 \mathrm{~g}$ ( $\mathrm{g}=$ gravitational acceleration). ${ }^{8}$ Previous studies demonstrated that LMHFV treatment could enhance muscle strength, ${ }^{6}$ postural control, ${ }^{9}$ balancing ability, ${ }^{6} 1011$ new bone formation, ${ }^{12-14}$ spinal $\mathrm{BMD}^{6}$ and blood circulation. ${ }^{6}$

There were some studies reporting the effect of wholebody vibration (WBV) treatment on wheelchair users with spinal cord injury (SCI). They showed that WBV treatment could increase upper limb performance during propulsion of the wheelchairs in terms of average speed, time of displacement and blood flow to paralysed muscles in the lower limbs. ${ }^{1516}$ However, there is a lack of evidence for the effect of LMHFV on older adults with poor walking and standing ability (such as non-paralysed wheelchair users), which is a missing gap of our knowledge.

In this study, we hypothesise that LMHFV would enhance the musculoskeletal health of the older adults with walking difficulties and relying on wheelchairs for daily mobility, in terms of vertebral bone quality and muscle performance. The objective is to investigate the effects of LMHFV on vertebral BMD, muscle performance and balancing ability in disabled older participants. The vibration platform will be modified to suit the disabled patients for treatment at sitting position. The results of this study would broaden the indications of vibration platform to benefit more people in the community, which have a good potential of translation.

\section{METHODS}

\section{Study design}

This is a single-blinded randomised controlled trial to study the effects of LMHV on vertebral BMD and muscle performance of disabled older adults (figure 1). This is a 2-year study in which the recruited wheelchair users will receive a 6-month LMHFV treatment. The study protocol was approved by the Clinical Research Ethics Committee of the Chinese University of Hong Kong (Ref. no 2019.087-T), with expected start date of January 1 of 2020 and end date of October 1 of 2021.

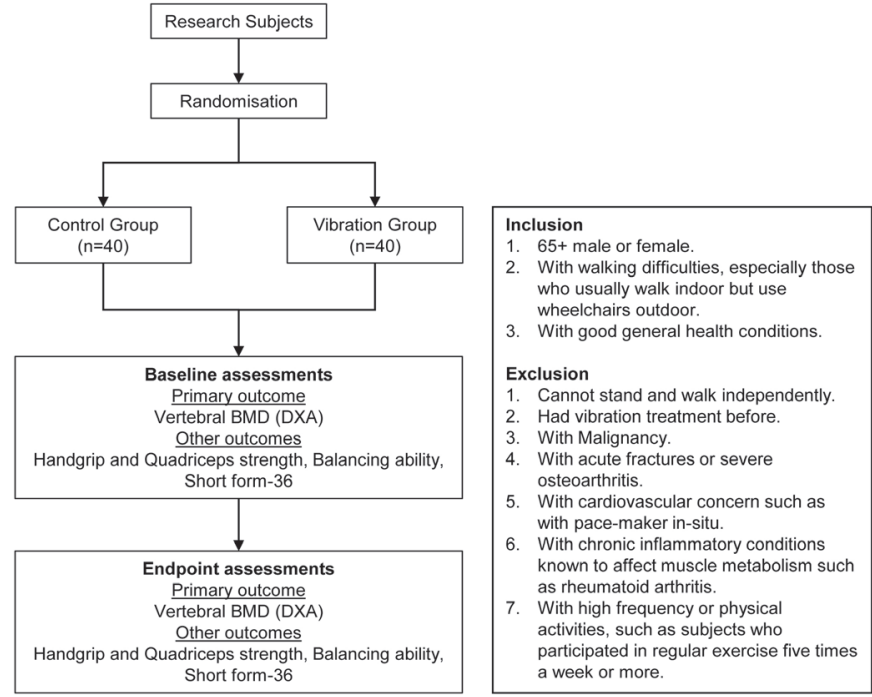

Figure 1 Flow diagram showing the grouping and assessments in this study. BMD, bone mineral density; DXA, dual-energy $\mathrm{X}$-ray absorptiometry.

\section{Study participants}

Participants will be recruited from the specialist outpatient clinic or orthopaedic wards of the Prince of Wales Hospital (PWH) of the Chinese University of Hong Kong, or community centres, or elderly homes. Participants will be recruited based on the inclusion and exclusion criteria. All recruited participants will be requested to give a written consent and will perform the following assessments at the PWH.

\section{Inclusion criteria}

Inclusion criteria are listed as follows:

1. Participants of both genders aged $\geq 65$ years.

2. Participants with walking difficulties, especially those who usually walk indoor but use wheelchairs outdoor.

3. Participants with good general health conditions.

\section{Exclusion criteria}

Exclusion criteria are as follows:

1. Participants cannot stand and walk independently.

2. Participants who had vibration treatment before.

3. Participants with malignancy.

4. Participants with acute fractures or severe osteoarthritis. $^{17}$

5. Participants with cardiovascular concern such as with pacemaker in situ.

6. Participants with chronic inflammatory conditions known to affect muscle metabolism such as rheumatoid arthritis.

7. Participants with high frequency of physical activities, such as participants who participated in regular exercise five times a week or more.

\section{Randomisation and grouping}

After eligibility screening, recruited participants will be randomised by sealed envelope drawing of computergenerated random numbers. The random number list is 
kept strictly confidential and the researchers will not have access to the list. Research participants will be randomised into either one of two groups: LMHFV or control group.

\section{Assessments}

Two groups of participants will be subjected to the same assessments: (1) demographic data collection; (2) BMD measurement at the spine (primary outcome) and hip; (3) balancing ability test; (4) quality of life (QoL) and (5) adverse event reporting, if any. Compliance of the participants in LMHFV group will be recorded in an Secure Digital (SD) card in the treatment device while the participants will also be given a treatment calendar to self-record the usage for cross-validation. Adverse or fall events are recorded by a calendar provided to each participant who will be instructed to record the date and details of each event as previously reported. ${ }^{6}$

\section{Interventions}

LMHFV is a non-invasive biophysical intervention providing WBV signals for mechanical stimulation. No known side effects are associated with this treatment except a small risk of falling during the transfer of participants from the wheelchair to the treatment device. As the benefits discussed above including muscle strength, ${ }^{6}$ postural control, ${ }^{9}$ balancing ability ${ }^{6} 1011$ and blood circulation $^{6}$ may benefit wheelchair users' musculoskeletal health, risks of falling could be minimised with the help of the on-site researchers.

Participants are required to sit upright on the vibration platform (V-Health, Hong Kong) providing a frequency $35 \mathrm{~Hz}, 0.3 \mathrm{~g}$ at peak-to-peak displacement of less than $0.1 \mathrm{~mm}$ for $20 \mathrm{~min} /$ day. Treatment will be given for at least 3 days/week. In this study, the design of vibration platforms is modified for wheelchair users. A chair and a

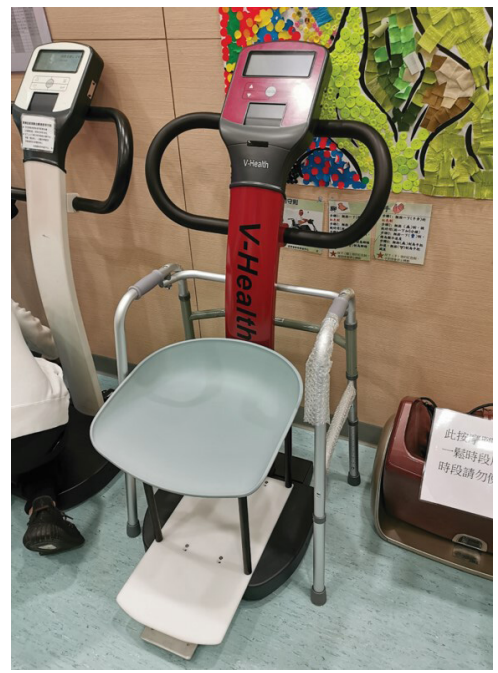

Figure 2 A vertical-oscillating vibration platform modified to provide a seating place for the research participant to receive the $20 \mathrm{~min} /$ day treatment. This representative vibration platform is installed in a community centre situated in a public housing estate with 6692 units/families in the Shatin district of Hong Kong. supporting footrest are fixed on the platform (figure 2). Participants are instructed to sit on the chair and rest their feet on the footrest for $20 \mathrm{~min} /$ day. The research staff will instruct safety precautions and operative procedures. Each patient in the control group is instructed to maintain their habitual lifestyle without vibration treatment or specific instructions given to take on additional physical exercise. Physical activities will be monitored via questionnaires.

\section{Blinding}

Investigators, outcome assessors and the statistician will be blinded to the grouping allocation that is performed by one independent staff and kept confidential from team members. Outcome assessments will be performed by a central technician at the Bone Quality and Health Centre of the Chinese University of Hong Kong, who has obtained International Society for Clinical Densitometry certificate and is independent from this project. All participants will be reminded not to disclose their grouping to the assessors. However, blinding the participants for the LMHFV treatment is not feasible because the vibration signals can be easily felt and placebo is rare in vibration studies. ${ }^{6} 10$

\section{Primary outcome measures}

All primary and secondary outcome assessments for the two groups will be performed at baseline and 6 months post treatment.

The primary outcome of this study is vertebral BMD. BMD will be assessed by standard dual-energy X-ray absorptiometry (DXA) (Delphi W, Hologic, Waltham, Massachusetts, USA), which is a gold standard assessment for osteoporosis recommended by the WHO. Spine and hip are the two standard sites for the diagnosis of osteoporosis and both will be measured. In our institute, the short-term precision error of areal BMD by DXA was $2.07 \%$ at the femoral neck and $1.35 \%{ }^{18}$ at the lumbar spine.

\section{Secondary outcome measures}

Secondary outcomes include muscle strength assessment, balancing ability and QoL.

Handgrip strength and quadriceps strength will be assessed. Handgrip strength will be measured by dynamometer (5030JI, JAMAR, USA) on dominant hand of each participant. Participants will be instructed to hold the device with the arm at right angle and elbow to the side of the body. The maximum effort will be taken from three trials. ${ }^{19}$ Quadriceps strength will be measured by instructing the participants to perform an active extension of the knee joint at a sitting position with both feet free from the ground, and the hip and knee joint flexed at $90^{\circ}$. The peak forces of the knee extension will be measured by a dynamometer attached at the malleoli level and repeated thrice in each lower limb with the maximum force taken for analysis. ${ }^{6}$

To assess the balancing ability, Modified Functional Reach Test ${ }^{20}$ and Postural Stability Test will be performed. Modified Functional Reach Test is a simple test for fall 
risk assessment. ${ }^{21}$ It measures the maximal distance that the participant can reach when the participant sits on a bench with their hip, knees and ankles positioned at $90^{\circ}$ of flexion, with feet positioned flat on the floor. A level yardstick will be mounted on the wall at the height of each participant's acromion of the non-paretic side, while sitting on bench with no back or arm rests. Participants are required to lean as far as possible in each direction without rotating or touching the wall. The furthest position of the fifth finger will be marked on the yardstick. If the participant cannot raise the paretic arm, the distance covered by the acromion during leaning will be used. ${ }^{17}$ Postural Stability Test measures participants' ability to maintain their centre of balance. Centre of pressure (COP) is the average point at which the body concentrates. COP can be tested by Biodex Balance System (Biodex Medical Systems, New York, USA). Participants are required to sit on the platform without support from feet and keep arms folded across chest. Thighs need to be kept parallel, with $75 \%$ of their length supported on the platform. ${ }^{22}$

To assess the health-related QoL, validated Chinese version of the 36-item Short-Form Health Survey will be used for quantitative evaluation of the participants' physical and mental component summary. Participants with higher scores indicate a general better QoL. ${ }^{63}$

\section{Sample size calculation}

Vertebral BMD is used as the primary outcome in this study. Our previous bed-rest study ${ }^{24}$ showed that vibration could reduce the loss of BMD at various skeletal sites by $5 \%-10 \%$. The total sample size is estimated to be 80 participants using two-way repeated measures ANOVA with power at 0.8 and alpha of 0.05 . With consideration of around $15 \%$ dropout, the sample size is increased to $\mathrm{n}=80$.

\section{Data analysis}

Data in this study will be analysed according to the intention-to-treat principle followed by per-protocol analysis. Missing data will be handled using last observation carried forward approach. Normality tests will be performed to determine the normal distribution of data. Two-way repeated measures ANOVA will be used for comparing the measured outcomes between two groups at different time points; $\chi^{2}$ tests are used to compare proportions for categorical variables. Non-parametric tests will be employed if normality assumption is violated. Additional subgroup analysis be explored and stratified by gender, Body Mass Index (BMI) and age. SPSS V.25.0 will be used to perform analysis, and significance level is set at $\mathrm{p}<0.05$.

\section{Data monitoring}

All investigators will be responsible for record keeping. Only principle investigator and co-investigators, authorised research personnel and ethical committee members can access the personal data during and after the study. All data collection will be performed with strict adherence to the professional standards of confidentiality. Any personal information, including participants' name, address, phone number, etc, will be removed from all records. Important documents will be retained for at least 3 years after the completion of the study for final report and inspection.

\section{Data statement}

Data and resources will be shared with other eligible investigators through academically established means. The protocol and datasets used or analysed in this study will be available from the corresponding author on reasonable request. Researchers who provide a methodologically sound proposal may access data to achieve aims in the approved proposal.

\section{Patient and public involvement}

Patients and the public were not involved in the design or planning of the study. Members of the public are involved in the recruitment of participants or conduct of the study. We will report test results to participants in plain language after their end-point assessments are completed. Results will be available to the public and patients in the forms of educational talks and booklets or flyers and published in open access peer-reviewed journals.

\section{Ethics and dissemination}

LMHFV is a non-invasive intervention and reported to have no serious adverse effect. ${ }^{6}$ The only ethical concern is the additional DXA assessments, which emits a low-dose radiation at $10 \mu \mathrm{Sv}$, roughly equivalent to 1 -day exposure to natural background radiation.

\section{DISCUSSION}

To our knowledge, this is the first study to investigate the efficacy of LMHFV therapy on wheelchair users. As the worldwide ageing population escalates, the number of people with mobility impairment or disability would also increase. ${ }^{25}$ These people would inevitably be relying on wheelchair to assist their daily living. ${ }^{26}$ Wheelchairbound elderlies usually have limited physical activity and are therefore associated with lower $\mathrm{BMD}^{27}$ and ability to control body position, and thus have an increased risk of fall and osteoporotic fractures. A study from Grant Medical Center in the USA investigated 30 wheelchairbound participants who fell from wheelchair over a 5-year period. ${ }^{28}$ The report showed that elderlies who were older than 65 years of age with higher fall risk were associated with lack of physical activity and poor nutrition, leading to a decrease in muscle mass and strength. As most osteoporotic fractures are caused by combination of poor balance, falls and deteriorating bone strength. Therefore, LMHFV is the proposed strategy to target musculoskeletal deterioration in wheelchair users for the prevention of more detrimental consequences.

LMHFV has previously been reported to have positive effects on reducing fall and fracture risks, enhancing 
muscle strength and improving balancing ability in healthy community-dwelling older people. ${ }^{629}$ Supported with this evidence, it is therefore hypothesised that LMHFV would provide positive effects on the musculoskeletal health and physical performance in terms of BMD, muscle strength and balancing ability on elderlies with disability or mobility impairments and who rely on wheelchairs for locomotion.

Vibration is a good treatment modality that is easy to implement on physically challenged patients, such as wheelchair users. However, there is a scarce of literature reporting its effect on this group of patients who are most desperately in need of the treatment. There have been a few reports on the application of vibration treatment on wheelchair users with SCI. Menéndez's study reported in 2016 investigated the acute effect of WBV. ${ }^{30}$ A total of 10 patients with SCI were recruited and treated with various treatment schemes including the WBV. Patients were instructed to sit on wheelchairs with their feet rested on the vibration platform (Galileo Home, Galileo, Novotec, Germany, $10 \mathrm{~Hz}, 5 \mathrm{~mm}$ peak-to-peak). Their results have shown an enhancement to the mean blood velocity and peak blood velocity by approximately $25 \%$ at $7 \mathrm{~min}$ after a 1-minute vibration treatment. A separate study in 2016, also by Menéndez's group, investigated the long-term combined treatment effect of WBV and electromyostimulatin (ES) in patients with SCI. A total of 17 participants with SCI were randomised into treatment group and control group for 12 weeks. Patients in treatment group received 30 sections of 10-minute WBV and ES stimulation over a 12-week period. ${ }^{31}$ The treatment group was reported to show increased resting arterial diameter, increased blood flow and increased muscle thickness at the gastrocnemius after 12 weeks of treatment. Although these studies employed a side-alternating vibration (instead of vertical-oscillating vibration) only to the lower limbs, these results suggested that mechanical stimulation in the form of vibration could provide benefits to blood flow that is associated with better performancerelated outcomes in paralysed skeletal muscles. Therefore, suggesting that the proposed LMHFV treatment, providing a vertical oscillating stimulation (figure 1) to the whole body, would be beneficial to the target group of patients.

Findings of the current clinical trial would generate valuable data that are of high clinical translation values for wider application of vibration therapy in the community, and to generate clinical evidence that is not restricted only to the healthy and mobile older people, but also to the group of patients with mobility impairments and high risk of further musculoskeletal function deterioration. Although the study only investigates a treatment period of 6 months due to limited research resources, a longer or sustained use of the vibration treatment may be observed as suggested by our previous study that beneficial effects on healthy older adults are maintained even after 12 months of treatment cessation. $^{29}$
Correction notice The author name Ronald Wong Man-Yeung Wong has been corrected to Ronald Man-Yeung Wong.

Acknowledgements We would like to thank all the colleagues in the Fall Prevention Team and the Bone Quality and Health Centre of the Department of Orthopaedics and Traumatology, Faculty of Medicine, The Chinese University of Hong Kong, for their support and help in this work and all the subjects for their participation in this study.

Contributors SKHC, YNC, RWM-YW and WHC conceptualised the study; SKHC, YNC and $\mathrm{CYH}$ drafted the manuscript and $\mathrm{CYH}$ and HWW executed the research project and data collection. All authors reviewed and approved the final manuscript.

Funding This study was supported by Project Impact Enhancement Fund (Ref: PIEF/U3/01), Evidence-Based Orthopaedics Clinical Education and Research Program (EBO) of the Chinese University of Hong Kong and V-Health Limited, by lending and modifying the vibration platforms used in this study.

\section{Competing interests None declared.}

Patient and public involvement Patients and/or the public were not involved in the design, or conduct, or reporting, or dissemination plans of this research.

Patient consent for publication Not required.

Provenance and peer review Not commissioned; externally peer reviewed.

Open access This is an open access article distributed in accordance with the Creative Commons Attribution Non Commercial (CC BY-NC 4.0) license, which permits others to distribute, remix, adapt, build upon this work non-commercially, and license their derivative works on different terms, provided the original work is properly cited, appropriate credit is given, any changes made indicated, and the use is non-commercial. See: http://creativecommons.org/licenses/by-nc/4.0/.

ORCID iDs

Yu Ning Chim http://orcid.org/0000-0002-6963-2538

Wing Hoi Cheung http://orcid.org/0000-0003-3247-8255

\section{REFERENCES}

1 Wong RMY, Choy MHV, Li MCM, et al. A systematic review of current osteoporotic metaphyseal fracture animal models. Bone Joint Res 2018;7:6-11.

2 Cole HA, Ohba T, Nyman JS, et al. Fibrin accumulation secondary to loss of plasmin-mediated fibrinolysis drives inflammatory osteoporosis in mice. Arthritis Rheumatol 2014;66:2222-33.

3 Chung S-L, Leung K-S, Cheung W-H. Low-magnitude highfrequency vibration enhances gene expression related to callus formation, mineralization and remodeling during osteoporotic fracture healing in rats. J Orthop Res 2014;32:1572-9.

4 Gebara OC, Mittleman MA, Sutherland P, et al. Association between increased estrogen status and increased fibrinolytic potential in the Framingham offspring study. Circulation 1995;91:1952-8.

5 Lindoff C, Petersson F, Lecander I, et al. Passage of the menopause is followed by haemostatic changes. Maturitas 1993;17:17-22.

6 Leung KS, Li CY, Tse YK, et al. Effects of 18-month low-magnitude high-frequency vibration on fall rate and fracture risks in 710 community elderly--a cluster-randomized controlled trial. Osteoporos Int 2014;25:1785-95.

7 Ghazalian F, Hakemi L, Pourkazemi L, et al. Effects of whole-body vibration training on fibrinolytic and coagulative factors in healthy young men. J Res Med Sci 2014;19:982-6.

8 Wang J, Leung KS, Chow SK, et al. The effect of whole body vibration on fracture healing - a systematic review. Eur Cell Mater 2017;34:108-27.

9 Rogan S, Hilfiker R, Herren K, et al. Effects of whole-body vibration on postural control in elderly: a systematic review and meta-analysis. BMC Geriatr 2011;11:72.

10 Cheung W-H, Mok H-W, Qin L, et al. High-frequency whole-body vibration improves balancing ability in elderly women. Arch Phys Med Rehabil 2007;88:852-7.

11 Torvinen S, Kannus P, Sievänen $\mathrm{H}$, et al. Effect of 8-month vertical whole body vibration on bone, muscle performance, and body balance: a randomized controlled study. J Bone Miner Res 2003;18:876-84.

12 Rubin C, Recker R, Cullen D, et al. Prevention of postmenopausal bone loss by a low-magnitude, high-frequency mechanical stimuli: a clinical trial assessing compliance, efficacy, and safety. J Bone Miner Res 2004;19:343-51. 
13 Verschueren SMP, Roelants M, Delecluse C, et al. Effect of 6-month whole body vibration training on hip density, muscle strength, and postural control in postmenopausal women: a randomized controlled pilot study. J Bone Miner Res 2004;19:352-9.

14 Ogborn DI, Gardiner PF. Effects of exercise and muscle type on BDNF, NT-4/5, and TrKB expression in skeletal muscle. Muscle Nerve 2010;41:385-91.

15 Wang X, Luo Y, Masci PP, et al. Influence of interleukin-1 beta on Platelet-Poor plasma clot formation: a potential impact on early bone healing. PLoS One 2016;11:e0149775.

16 Francis RM, Romeyn CL, Coughlin AM, et al. Age and aerobic training status effects on plasma and skeletal muscle tPA and PAI-1. Eur J Appl Physiol 2014;114:1229-38.

17 Choi S-J, Shin W-S, Oh B-K, et al. Effect of training with whole body vibration on the sitting balance of stroke patients. J Phys Ther Sci 2014;26:1411-4.

18 Zhu TY, Hung VWY, Cheung W-H, et al. Value of measuring bone microarchitecture in fracture discrimination in older women with recent hip fracture: a case-control study with HR-pQCT. Sci Rep 2016;6:34185.

19 Wong RMY, Wong $\mathrm{H}$, Zhang $\mathrm{N}$, et al. The relationship between sarcopenia and fragility fracture-a systematic review. Osteoporos Int 2019;30:541-53.

20 Gao KL, Chan KM, Purves S, et al. Reliability of dynamic sitting balance tests and their correlations with functional mobility for wheelchair users with chronic spinal cord injury. J Orthop Translat 2015;3:44-9.

21 Williams B, Allen B, Hu Z, et al. Real-time fall risk assessment using functional reach test. Int J Telemed Appl 2017;2017:2042974

22 Ruhe A, Fejer R, Walker B. Center of pressure excursion as a measure of balance performance in patients with non-specific low back pain compared to healthy controls: a systematic review of the literature. Eur Spine J 2011;20:358-68.

23 Lam CL, Gandek B, Ren XS, et al. Tests of scaling assumptions and construct validity of the Chinese $(\mathrm{HK})$ version of the SF-36 health survey. J Clin Epidemiol 1998;51:1139-47.

24 Wang H, Wan Y, Tam K-F, et al. Resistive vibration exercise retards bone loss in weight-bearing skeletons during 60 days bed rest. Osteoporos Int 2012;23:2169-78.

25 Mann WC, Llanes C, Justiss MD, et al. Frail older adults' self-report of their most important assistive device. OTJR 2004;24:4-12.

26 Gavin-Dreschnack D, Nelson A, Fitzgerald S, et al. Wheelchairrelated falls: current evidence and directions for improved quality care. J Nurs Care Qual 2005;20:119-27.

27 Cavedon V, Milanese C, Laginestra FG, et al. Bone and skeletal muscle changes in oldest-old women: the role of physical inactivity. Aging Clin Exp Res 2020;32:207-14.

28 Opalek JM, Graymire VL, Redd D. Wheelchair falls: 5 years of data from a level I trauma center. J Trauma Nurs 2009;16:98-102.

29 Cheung W-H, Li C-Y, Zhu TY, et al. Improvement in muscle performance after one-year cessation of low-magnitude highfrequency vibration in community elderly. J Musculoskelet Neuronal Interact 2016;16:4-11.

30 Menéndez H, Ferrero C, Martín-Hernández J, et al. Chronic effects of simultaneous electromyostimulation and vibration on leg blood flow in spinal cord injury. Spinal Cord 2016;54:1169-75.

31 Herrero AJ, Menéndez H, Gil L, et al. Effects of whole-body vibration on blood flow and neuromuscular activity in spinal cord injury. Spinal Cord 2011;49:554-9. 